\title{
An effective pressure-flow characterization of respiratory asynchronies in mechanical ventilation
}

\author{
Alberto Casagrande • Francesco \\ Quintavalle · Rafael Fernandez • Lluis \\ Blanch • Massimo Ferluga • Enrico \\ Lena - Francesco Fabris • Umberto \\ Lucangelo
}

Received: 13 August 2019 / Accepted: 22 January 2020

\begin{abstract}
Purpose: Ineffective Effort during Expiration (IEE) occurs when there is a mismatch between the demand of a mechanically ventilated patient and the support delivered by a Mechanical Ventilator (MV) during the expiration. This work presents a pressure-flow characterization for respiratory asynchronies and validates a machine-learning method, based on the presented characterization, to identify IEEs.
\end{abstract}

This work is partially supported by INdAM/GNCS. Lluis Blanch is supported in part by Plan Avanza TSI-020302-2008-38, Ministerio de Industria, Turismo y Comercio (Spain) and Ministerio de Ciencia, Innovación y Universidades (Spain).

Alberto Casagrande $(\varangle)$. Francesco Fabris

Dept. Mathematics and Geosciences

University of Trieste

Via Valerio, 12/1, I-34127 Trieste - ITALY

E-mail: acasagrande@units.it

Tel.: +390405582620

Francesco Quintavalle · Massimo Ferluga · Enrico Lena .

Umberto Lucangelo

DAI Emergenza Urgenza ed Accettazione

Azienda Sanitaria Univeritaria integrata di Trieste,

Trieste - ITALY

Lluis Blanch

Critical Care Center

ParcTaulì Hospital Universitari,

Institut d'Investigaciò i Innovaciò Parc Taulì I3PT,

Universitat Autònoma de Barcelona,

Barcelona - SPAIN

CIBER Enfermedades Respiratorias,

Instituto de Salud Carlos III, Madrid - SPAIN

Rafael Fernandez

CIBER Enfermedades Respiratorias,

ICU, Hospital Sant Joan de Déu,

Fundació Althaia, Manresa - SPAIN 
Methods: 1500 breaths produced by 8 mechanically-ventilated patients were considered: 500 of them were included into the training set and the remaining 1000 into the test set. Each of them was evaluated by 3 experts and classified as either normal, artefact, or containing inspiratory, expiratory, or cycling-off asynchronies. A software implementing the proposed method was trained by using the experts' evaluations of the training set and used to identify IEEs in the test set. The outcomes were compared with a consensus of three expert evaluations.

Results: The software classified IEEs with sensitivity 0.904 , specificity 0.995 , accuracy 0.983, Positive (PPV) and Negative Predictive Value (NPV) 0.963 and 0.986 , respectively. The Cohen's kappa is 0.983 (with $95 \%$ Confidence Interval (CI): $[0.884,0.962])$.

Conclusion: The pressure-flow characterization of respiratory cycles and the monitoring technique proposed in this work automatically identified IEEs in real-time in close agreement with the experts.

Keywords Respiratory asynchrony $\cdot$ mechanical ventilator $\cdot$ automatic monitoring $\cdot$ machine learning

\section{Introduction}

Respiratory asynchrony is a condition experienced by subjects that suffer from limited autonomous respiratory capability and receive ventilation support that does not match their requests in terms of duration, volume and flow. Such poor patient-ventilator interaction affects up to $43 \%$ of mechanically ventilated patients 1,2,3,4 and has been associated with several undesirable phenomena such as dyspnea $5,6,7,8,9$, increased duration of mechanical ventilation 1, 10, raised need of sedative and paralytic agents [11,12,13, need for tracheostomy [1, respiratory muscle injury [14,15, 16, and mortality [17. Ineffective efforts during expiration (IEE), double triggering and auto-triggering are the most common patient-ventilator asynchronies [1,18.

Asynchronies are usually detected by observing a patient's bedside timeflow and time-pressure graphs. However, given the large variability of asynchronies, the problem is often under-diagnosed due to the difficulty in their recognition and shortage of specialists $[19$.

Oesophageal pressure waveform can also be used to detect asynchronies, but it is semi-invasive and requires strict calibration, the accuracy of the signal is affected by several variables (e.g., patient's posture, cardiac activity, swallowing, position and volume of inflation of the oesophageal balloon) and specific skills for the interpretation of tracings are required [20,21. Electrical Activity of diaphragm signal (EAdi) can be used either for monitoring patient-ventilator interaction 22 and for setting up Neurally Adjusted Ventilatory Assist $\left(\mathrm{NAVA}^{\circledR}{ }^{\circledR}\right)$; similarly to oesophageal pressure, careful nasogastric catheter (EAdi catheter) positioning is needed. Moreover, patients can trigger ventilators by using solely the auxiliary respiratory muscles: this means, according to "first-come, first-served" principle, that even during NAVA ${ }^{\circledR}$ there 
may be spontaneous ventilatory activity, and therefore asynchrony, even in the absence of any EAdi evidence because, in the specific case, software switches to an actual PSV (Pressure Support Ventilation) with a non-diaphragmatic pneumatic trigger $23,24,25$. The relevance of the problem suggests the need for an automatic monitoring system able to recognize asynchronies and alert clinicians. Some dedicated techniques and software have already been developed to achieve this. Regrettably, their performance is not comparable to that of a human expert as well as exhibiting limitations in terms of usage [3,4,26, 27, 28.

This manuscript presents a new characterization for asynchronies: they are seen as work overloads which may produce small loops in the pressureflow representation of breaths. The suggested approach phases out the need for EAdi and oesophageal pressure analysis during asynchrony identification and allows to exclusively focus on flow and pressure evolution. This work also introduces a method to detect IEEs by targeting and analyzing small loops and compares its effectiveness and that of a pool of experts in the field.

The characterization of respiratory asynchronies investigated by this article is part of the EU patent "Apparatus to Identify Respiratory Asynchronies studied in an Assisted Breathing Machine" No. EP 3308819 (A1) [29].

\section{Materials and Methods}

\subsection{Asynchronies as Work Overloads}

In assisted respiratory cycles, patients and MVs interact as a single system and, whenever they are out-of-sync -because the patient either inhales while the ventilator pulls air or exhales while the ventilator pumps air into their lungs-, a deflection on both time-flow and time-pressure graphs occurs (e.g., see Fig. 11. Tipically, asynchronies have been identified by human experts on the basis of the deflection type.

From a physical point of view, any asynchrony is a work overload for the overall patient-ventilator system due to impedance in the respiratory activity.

Campbell [30,31] observed that any respiratory cycle corresponds to a loop cycle in the pressure-volume space and the area of the latter measures the work of breath (WOB) needed to produce the former. This consideration can be shifted in the pressure-flow space by observing that a constant flow $(f)$ and the corresponding volume $(V)$ are related on the basis of the equation $V=f * \delta_{t}$ where $\delta_{t}$ stands for the time interval. Hence, any loop in the pressure-volume space is connected to a loop in the pressure-flow space whose area corresponds to the power of breathing $(P)$ and can be computed as

$$
P=\int f(p) d p
$$

where $p$ is the pressure. Moreover, if $\delta_{t}$ is constant in time -and this is the case for data produced by MVs-, it also holds that $\Delta \mathrm{WOB}=P * \delta_{t}$. Hence, 

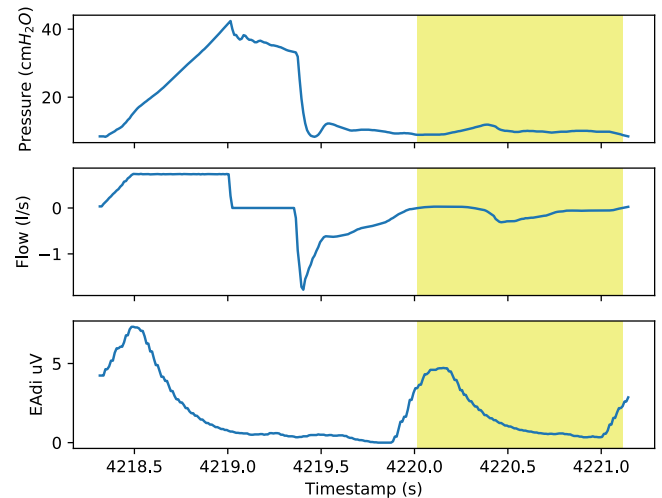

Fig. 1: The pressure-time, flow-time, and EAdi-time representation of a breath containing an IEE. IEE corresponds to a deflection on flow and pressure waveforms between timestamps $4220.0 \mathrm{~s}$ and $4221.1 \mathrm{~s}$. In this case, EAdi has a peak during the deflection around timestamp $4220.1 \mathrm{~s}$. In the figure, the coloured region highlights the time interval during which the deflection itself occurs.

whenever a smaller loop decorates the breath loop cycle, the former represents a work overload which is measurable by computing its area (e.g., see Fig. 2). Such small loops are named sub-breath loops (SBLs).

Even though the presence of a SBL in the pressure-flow space guarantees that the patient-ventilator system has experienced a work overload, not all work overloads produce SBLs in the same space. Moreover, work overloads are not always due to asynchronies and can occur in different situations such as, for instance, when a patient expectorates.

We observed that this miss-correspondence between SBLs and work overload occurs in particular during inspiration and, because of that, despite also different types of asynchrony (i.e. inspiratory and cycling-off asyncrony) were initially considered, this preliminary work then focused exclusively on IEE detection.

\subsection{A New IEE Identification Algorithm}

The characterization proposed by Section 2.1 suggests a two-step algorithm to label respiratory cycles as either normal or containing IEEs. The first step consists in identifying all the SBLs in the pressure-flow representation of a breath. If the breath does not exhibit any of them, then it is labelled as normal. Otherwise, the second step classifies the SBLs as either associated to IEE or "spurious loops", i.e., not related to any IEE, by means of a machine-learning technique based on geometric features of the SBLs themselves. Whenever a 


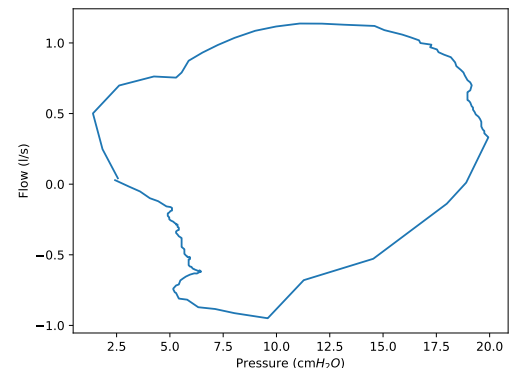

(a) A normal breath

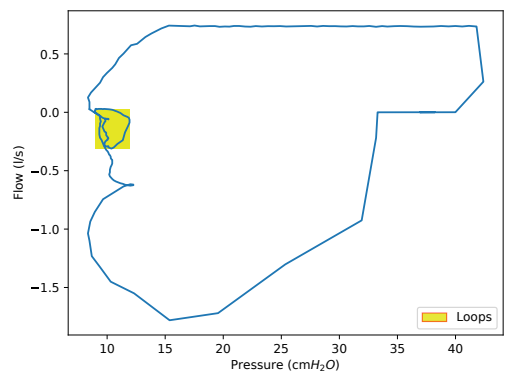

(b) A breath containing an IEE in the form of SBL

Fig. 2: Two respiratory cycles portrayed in pressure-flow space: Fig. 2a represents a normal breath, while Fig. 2b contains an IEE which is associated to a sub-breath loop (SBL) clearly identifiable inside the coloured rectangle. Fig. 1 and Fig. $2 \mathrm{~b}$ depicts the same respiratory cycle and the SBL highlighted in the latter corresponds to the deflection emphasized by using the same colour in the former.

SBL in a breath is classified as corresponding to an IEE, the breath itself is labelled as containing IEEs.

\subsection{Data Collection}

We retrospectively analyzed data collected for a previously published study [27. Flow $(F)$, airways pressure $(p)$ and EAdi samples for a total of 50228 breaths by eight critically ill mechanically ventilated patients were considered.

The patients were ventilated with Servo-i ${ }^{\circledR}$ (Maquet) in different modalities: Pressure Regulated Ventilation Controlled (PRVC), Pressure Support (PS), Synchronized Intermittent Mandatory Ventilation-Ventilation Controlled (SIMV-VC), Synchronized Intermittent Mandatory Ventilation-Pressure Control (SIMV-PC), Volume Control (VC), Volume Support (VS), and NAVA ${ }^{\circledR}$.

Full details on inclusion and exclusion criteria, informed consent, Ethics Committee approval, study registration, study location, as well as the start and the end date of inclusion can be found in [27].

\subsubsection{Expert Classifications}

Two disjoint sets of 500 and 1000 breaths were randomly selected among the considered 50228 respiratory cycles. The breaths in the former set were used to train the SBL classifier. These cycles were collegially evaluated by three experts in mechanical ventilation and patient-ventilator asynchronies (the authors L.B., R.F., and E.L.) in order to maximize the training accuracy. Con- 
versely, the latter set was adopted as the test set for the overall classification process and its breaths were individually evaluated by a team of three experts different from those who curated the training set (L.B., R.F., and M.F.). In this manner, a clinical environment was simulated where a single physician gauged the respiratory cycles of patients.

The 1500 breaths were shuffled and each of them was presented as a figure containing the pressure-time, flow-time, and EAdi-time evolution of the breath itself (e.g., see Fig. 1). A breath could be classified as either normal, containing respiratory asynchronies, or presenting artefacts -which may be due to spurious measurements rather than asynchronies-. Whenever a breath was classified as containing respiratory asynchronies, the experts further specified whether the asynchronies were inspiratory, expiratory, or cycling-off; one or more types of asynchronies could be selected for a single breath.

The results of these evaluations were a single classification for each cycle in the training set -due to the collegial evaluations of the experts- and three distinct classifications for each of the breaths in the test set.

\subsection{Implementation of the Proposed Procedure}

A software prototype which implements the proposed procedure was coded in Python. The SBL identification procedure and the features extraction were coded in plain Python. On the contrary, the process to automatically gather the SBL classifier from the training set -i.e., the training phase- and the SBL classifier itself also took advantage of NumPy [32, Pandas [33,34, and scikit-learn [35] libraries.

\subsection{SBL Identification and Features Extraction}

The first step of the proposed algorithm considers flow and pressure samples of a single breath at a time. The pairs of subsequent samples are coupled and segments in pressure-flow space are built; the time order of the samples is inherited by these segments. Finally, all the intersections were spotted: the values in the segment sequence between two intersecting segments form a loop.

In order to select SBLs, all the loops lasting either less than $0.1 \mathrm{~s}$ or more than $3.0 \mathrm{~s}$ are arbitrary discharged. The former are most likely due to measurement disturbances, the latter are more likely to be complete respiratory cycles. Moreover, since the aim is to both reduce computational time and find significant IEEs, the SBLs whose area feature is below $8 \times 10^{-3} \mathrm{cmH}_{2} \mathrm{Ol} / \mathrm{s}$ (i.e., $\approx 7.84 \times 10^{-4} \mathrm{~J} \mathrm{~s}^{-1}$ ) are also filtered out.

Once a SBL has been identified, the software computes its features to establish whether it corresponds to an IEE. Different traits were considered to achieve this goal and the scikit-learn's selector SelectFromModel (see [36] and [37 for more details about scikit-learn's API) was used to choose the most effective features to classify SBLs in the training set by using logistic regression (see Table 1). 
Table 1: These features are used to classify loops as either associated to an IEE or spurious loop. All of them, except the last two -i.e., switch time and ventilation mode-, were selected from the scikit-learn's feature selector feature_selection.SelectFromModel as the most significant ones to classify the loops in the training set by using logistic regression. The switch time feature exclusively helps in distinguishing expiration loops from the inspiration ones, while the ventilation mode feature is used to provide a ventilation mode-specific classification. It ought to be noted that, while some of them are related, -e.g., length ratio, \# of insp. samples, and length-, they are linearly independent.

\begin{tabular}{|c|c|}
\hline begin time & $\begin{array}{l}\text { time elapsed between the breath begin time and the loop } \\
\text { begin time }\end{array}$ \\
\hline length & time frame of the loop data-set \\
\hline area & area of the loop in the pressure-flow space \\
\hline work & $\begin{array}{l}\text { work overload associated to the loop and computed as } \\
\int_{s \in \text { samples }} p_{s} * f_{s} d t_{s}\end{array}$ \\
\hline bounding box area & $\begin{array}{l}\text { area of the smallest rectangle in the pressure-flow space } \\
\text { containing the loop }\end{array}$ \\
\hline maximum flow & $\begin{array}{l}\text { maximum flow value among the samples participating } \\
\text { to the loop }\end{array}$ \\
\hline \# of insp. samples & $\begin{array}{l}\text { number of samples in the inspiratory phase preceding } \\
\text { the loop }\end{array}$ \\
\hline length ratio & $\begin{array}{l}\text { ratio between the begin feature and time frame of the } \\
\text { whole respiratory cycle in which the loop occurs }\end{array}$ \\
\hline begin ratio & $\begin{array}{l}\text { ratio between the begin feature and time frame of the } \\
\text { whole respiratory cycle in which the loop occurs }\end{array}$ \\
\hline switch pressure & $\begin{array}{l}\text { pressure value in the first sample of the expiratory phase } \\
\text { in which the loop occurs }\end{array}$ \\
\hline switch flow $\delta$ & $\begin{array}{l}\text { difference between the flow value at the beginning of the } \\
\text { expiratory phase and that in the first sample of the loop }\end{array}$ \\
\hline switch pressure $\delta$ & $\begin{array}{l}\text { difference between the pressure value at the beginning } \\
\text { of the expiratory phase and that in the first sample of } \\
\text { the loop }\end{array}$ \\
\hline switch time & $\begin{array}{l}\text { time elapsed between the breath begin time and the } \\
\text { switch from inspiration to expiration phase }\end{array}$ \\
\hline ventilation mode & $\begin{array}{l}\text { MV operating mode: NAVA }{ }^{(\mathbb{R})} \text {, PRVC, PS, SIMV-PC, } \\
\text { SIMV-VC, or VC }\end{array}$ \\
\hline
\end{tabular}

The patient's respiratory phase is inferred from the data supplied by the MV. Each sample reports the ventilator respiratory phase which, from time to time, is quickly switched from inspiration to expiration and back in an attempt to equalize patient demand and ventilator supply. We discretionarily consider 20 samples in a row reporting the same phase for MV to be a clue that the patient is going through that phase too. By making this assumption, the software is able to both label SBLs as either inspiratory or expiratory events and filter the former as not useful for identifying IEEs.

The SBL features are also enriched with the ventilation mode-i.e., NAVA ${ }^{\circledR}$, PRVC, PS, SIMV-PC, SIMV-VC, VC, or VS- provided by the MV. This 
helped in establishing whether the traits of IEE SBLs are dependent on the MV operating mode and, if this is the case, in providing a mode specific classification.

\subsubsection{Training the SBL Classifier}

The experts' evaluations and the loops identified by our software were paired by using a Python script. In order to deal with breaths that were labelled as containing IEEs by the experts and exhibited more than one expiratory SBL, we arbitrary decided to exploit also the EAdi data and associate the IEE labels only to those expiratory SBLs that have the greatest EAdi in their breath. EAdi was not used during any subsequent classification.

The loops were partitioned according to the ventilation mode reported by the MV during the loop itself and seven different classifiers, $C_{\mathrm{SIMV}-\mathrm{PC}}$, $C_{\mathrm{SIMV}-\mathrm{VC}}, C_{\mathrm{NAVA}}{ }^{\circledR}, C_{\mathrm{PS}}, C_{\mathrm{PRVC}}, C_{\mathrm{VS}}$, and $C_{\mathrm{VC}}$, were trained by using linear logistic regression as implemented by the class LogisticRegression in the scikit-learn library. The features presented in Table 1 were automatically scaled by scikit-learn's StandardScaler and used as classifier inputs. SBL classifications and, thus, classifier outputs were encoded in binary values: 1 if the considered SBL was associated to an IEE, 0 otherwise. The class SelectFromModel was also used to filter the irrelevant features and reduce the classifier input size.

For any set of features, the classifiers return the probability that those features have to be related to an IEE loop. Probabilities greater than 0.5 were interpreted as "the features come from an IEE loop", while those smaller or equal to 0.5 as "the features come from a spurious loop".

A global classifier $C_{S}$ was then built by composing all the mode-specific classifiers: for each SBL to be evaluated, the opportune classifier is selected among those ventilation specific by using the ventilation mode feature; this classifier evaluates the odds for the loop to be related to an IEE.

\subsection{Testing the Approach}

A software pipeline, consisting of the SBL identification, the features extractor and the SBL classifier, was applied to the breaths in the test sets and an automatic classification for each of the discovered SBLs was produced. This naturally induced a classification also for the breaths: every breath containing at least one IEE loop is is labelled as containing IEEs.

In order to analyze the differences between the automatic classifications of breaths and those due to the experts, a consensus of the latter was assembled. In particular, only those cycles on which at least two experts yield the very same evaluation were considered. The classifications for these respiratory cycles were synthesized by majority and compared to those output by the pipeline. 


\section{Results}

According to the experts, 69 breaths, among the 500 in the training set, contained IEEs. All the breaths in this set that contained IEEs exhibited at least one SBL during the expiratory phase and 11 of them had more than one SBL (see Table 2 for more details).

Table 2: Statistics from the training set referring to the experts' classifications (either IEEs or No IEEs) and MV ventilation mode. Since the proposed algorithm tags as not affected by IEEs all the breaths lacking expiratory SBLs and leaves the remaining ones to the judgment of the SBL classifier, the table distinguishes the classifications for former (columns "With SBLs") from those for latter (columns "No SBLs"). None of the breaths lacking expiratory SBLs was classified as affected by IEEs by the experts (column "IEEs" under "No SBLs"). The training set did not contain any breath produced in VS.

\begin{tabular}{lrrrrrrr}
\hline & \multicolumn{2}{c}{ With SBLs } & & \multicolumn{2}{c}{ No SBLs } & \\
\cline { 2 - 3 } V. Mode & IEEs & No IEEs & & IEEs & No IEEs & Total \\
\hline PS & 16 & 75 & & 0 & 109 & 200 \\
SIMV-VC & 8 & 10 & & 0 & 128 & 146 \\
NAVA & 1 & 11 & & 0 & 45 & 57 \\
SIMV-PC & 10 & 8 & & 0 & 24 & 42 \\
PRVC & 33 & 1 & & 0 & 1 & 35 \\
VC & 1 & 18 & & 0 & 1 & 20 \\
VS & 0 & 0 & & 0 & 0 & 0 \\
\hline Total & 69 & 123 & & 0 & 308 & 500 \\
\hline
\end{tabular}

The three experts that evaluated the test set (1000 breaths) fully agreed only on 182 respiratory cycles, while the consensus, which was produced by considering only those cycles on which at least two experts fully agreed on the classification, accounted for 903 breaths.

None of the SBLs in the training set occurred in the VS mode and, because of this, it was not possible to train the $C_{\mathrm{VS}}$ classifier. As result $C_{S}$ is not able to identify IEEs in VS at the moment. Unfortunately, one the breaths in the test set that contains a SBL was controlled by this mode and, thus, we decided to label it as "not classifiable".

The training set and the test sets contained 1500 breaths and consisted of about 4897 seconds of total recordings. The SBL identification and the feature extraction took 179 seconds -i.e., 2 minutes and 59 seconds- to process all of them on a Intel@ Core $^{\mathrm{TM}} \mathrm{i} 7-6700 \mathrm{HQ} \mathrm{CPU}$ at $2.60 \mathrm{GHz}$ and a GeForceß GTX $960 \mathrm{M}$ with 2GB of RAM; it was able to identify 223 expiratory SBLs in 192 cycles among the 500 breaths of the training set and 351 expiratory SBLs in 302 different respiratory cycles among the 1000 breaths in the test set. 
The training phase required, on the same hardware, less than 0.1 seconds to build the overall $C_{S}$ classifier and the subsequent classification of the SBLs in the test sets took about the same amount of time.

The proposed method tagged as normal 11 breaths among the 114 containing IEEs, while it correctly labelled 784 cycles among the 789 that do not contain IEEs (see Table 3 for more details). It follows that its sensitivity is about 0.904 (with $95 \%$ CI is $[0.834,0.951]$ ), its specificity 0.995 (95\% $\mathrm{CI}$ is $[0.987,0.999])$, its positive $(\mathrm{LR}+)$ and negative likelihood ratios (LR-) 177.991 and 0.097 , respectively $(95 \% \mathrm{CI}$ are $[66.85,473.94]$ and $[0.06,0.17]$, respectively), its accuracy 0.983 (95\% CI is [0.973, 0.991]), its PPV and NPV 0.963 and 0.986 , respectively $(95 \% \mathrm{CI}$ are $[0.906,0.986]$ and $[0.976,0.992]$, respectively), and its Cohen's kappa is about 0.983 (95\% CI is [0.884, 0.962]).

\section{Discussion and Conclusions}

All the considered indexes highlight the strength of the proposed technique and advocate in favour of its adoption in clinical environments as a support tool to automatically identify IEEs and, if needed, to alert physicians.

While a direct comparison on the very same data set is required to establish relative effectiveness, a rough observation of the data published in [27] suggests that the proposed approach has a higher specificity with respect to Better Care ${ }^{\circledR}$, the leading software for IEE automatic identification. Consequently, the PPV and Cohen's Kappa of the former are also higher than those of the latter. This is even more impressive in light of four main observations.

First of all, our classification pipeline does not take advantage of EAdi unlike the experts who had the chance to do so. We used EAdi exclusively during the training phase which was performed once for all and no longer requires re-running. The presented results seem to suggest that this signal is not needed to provide an accurate identification of IEEs.

Secondly, the timing collected during tests certifies that, although the pipeline is a prototype written in a programming language not known for its efficiency (e.g., see [38]), it can analyze respiratory cycles in real-time and still maintain good accuracy. This is not always true for human experts since they may spend a significant amount of time evaluating controversial cases. The small number of respiratory cycles in the test set that receive identical evaluation from the three experts -i.e., 182 among 1000 breaths- vouches to how difficult is for clinicians to diagnose respiratory asynchronies despite a potentially unbounded amount of time and the availability of EAdi. Moreover, Vaporidi et al. pointed out the importance of variability of IEEs occurrence over time rather than their mere incidence (i.e., Asynchrony Index, which can only be identified retrospectively) in the same patient in terms of duration of mechanical ventilation and hospital mortality, suggesting the need for realtime analysis of breaths 26$]$.

Thirdly, the lack of expiratory SBLs seems to be a sufficient condition to guarantee the absence of IEEs (see Table 3 columns "No SBLs"). This testifies 
Table 3: Some statistics about the test set. By using the experts' classifications as a reference standard, we labelled each automatic classification as true positive (TP), false positive (FP), false negative $(\mathrm{FN})$, or true negative $(\mathrm{TN})$ if it reported the presence of IEEs as the experts did, if it reported the presence of IEEs differently from what the experts did,if it did not identify any IEE whereas the experts did so, or if it did not identify any IEE consistently with the experts' classification, respectively. Each row of the table corresponds to a ventilation mode and reports the number of TP, FP, FN, and TN classifications about breaths in the consensus of the test set. Similarly to Table 2 , this table distinguishes between breaths exhibiting expiratory SBLs (columns "With SBLs") and breaths lacking expiratory SBLs (columns "No SBLs"). It should be pointed out that the breaths that were missing expiratory SBLs were always evaluated as not exhibiting IEE by the proposed procedure, thus, neither TP nor FP classifications are produced for this kind of breaths and thus missing from the report. The number of the FN and TN classifications of the proposed procedure are the sums of the corresponding values in the columns With $S B L s$ and No $S B L$. As mentioned in the text, $C_{\mathrm{VS}}$ was not trained because the training set did not contain any breath in VS. Unfortunately, one such breath was present in the consensus and, because of this, was labelled as not classifiable (NC).

\begin{tabular}{|c|c|c|c|c|c|c|c|c|c|c|}
\hline \multirow[b]{3}{*}{ V. Mode } & \multicolumn{8}{|c|}{ In Consensus } & \multirow{3}{*}{$\begin{array}{c}\text { Not in } \\
\text { Consensus }\end{array}$} & \multirow[b]{3}{*}{ Total } \\
\hline & \multicolumn{5}{|c|}{ With SBLs } & \multicolumn{2}{|c|}{ No SBLs } & \multirow[b]{2}{*}{ Total } & & \\
\hline & $\mathrm{TP}$ & FP & $\mathrm{FN}$ & $\mathrm{TN}$ & $\mathrm{NC}$ & FN & $\mathrm{TN}$ & & & \\
\hline PS & 25 & 3 & 7 & 119 & 0 & 0 & 212 & 366 & 30 & 396 \\
\hline SIMV-VC & 13 & 1 & 2 & 11 & 0 & 0 & 249 & 276 & 22 & 298 \\
\hline NAVA ${ }^{\circledR}$ & 0 & 0 & 1 & 20 & 0 & 0 & 82 & 103 & 5 & 108 \\
\hline SIMV-PC & 19 & 0 & 0 & 12 & 0 & 0 & 52 & 83 & 9 & 92 \\
\hline PRVC & 46 & 0 & 1 & 1 & 0 & 0 & 1 & 49 & 21 & 70 \\
\hline $\mathrm{VC}$ & 0 & 0 & 0 & 20 & 0 & 0 & 5 & 25 & 9 & 34 \\
\hline VS & 0 & 0 & 0 & 0 & 1 & 0 & 0 & 1 & 1 & 2 \\
\hline Total & 103 & 4 & 11 & 183 & 1 & 0 & 601 & 903 & 97 & 1000 \\
\hline
\end{tabular}

the effectiveness of the proposed characterization for expiratory asynchronies. It remains to be investigated whether an analogous condition also holds during the inspiration phase.

Finally, the SBL classifier gives proof of good performances despite the small cardinality of the training set and its simplicity -it is a linear model-. We believe that considering more SBLs for the training set and re-implementing the classifier itself by means of a more advanced machine learning technique can further improve the classification outcomes. We plan to investigate this aspect as well as the applicability of the characterization proposed in this work to the identification of both inspiratory and cycling-off asynchronies in the near future. 


\section{Conflicts of interest}

Lluis Blanch is inventor of one Corporació Sanitaria Parc Taulí owned US patent: "A Method and system for managed related patient parameters provided by a monitoring device", US Patent No. 12/538,940. Lluis Blanch is a founder of BetterCare S.L.: a research and development company, spin-off of Corporació Sanitària Parc Taulí. Lluis Blanch is supported in part by Plan Avanza TSI-020302-2008-38, MCYIN and MITYC (Spain). Umberto Lucangelo own stock options of BetterCare S.L. Alberto Casagrande, Francesco Quintavalle, Francesco Fabris, and Umberto Lucangelo are inventors of the EU patent "Apparatus To Identify Respiratory Asynchronies In An Assisted Breathing Machine" European Patent no. EP 3308819 (A1) 29].

\section{References}

1. Thille, A.W., Rodriguez, P., Cabello, B., Lellouche, F., Brochard, L.: Patient-ventilator asynchrony during assisted mechanical ventilation. Intensive care medicine 32(10), 1515-1522 (2006)

2. Vignaux, L., Vargas, F., Roeseler, J., Tassaux, D., Thille, A.W., Kossowsky, M.P., Brochard, L., Jolliet, P.: Patient-ventilator asynchrony during non-invasive ventilation for acute respiratory failure: a multicenter study. Intensive care medicine 35(5), 840-846 (2009)

3. Chen, C.W., Lin, W.C., Hsu, C.H., Cheng, K.S., Lo, C.S.: Detecting ineffective triggering in the expiratory phase in mechanically ventilated patients based on airway flow and pressure deflection: Feasibility of using a computer algorithm*. Critical care medicine 36(2), 455-461 (2008)

4. Mulqueeny, Q., Ceriana, P., Carlucci, A., Fanfulla, F., Delmastro, M., Nava, S.: Automatic detection of ineffective triggering and double triggering during mechanical ventilation. Intensive care medicine 33(11), 2014-2018 (2007)

5. Schmidt, M., Demoule, A., Polito, A., Porchet, R., Aboab, J., Siami, S., Morelot-Panzini, C., Similowski, T., Sharshar, T.: Dyspnea in mechanically ventilated critically ill patients*. Critical care medicine 39(9), 2059-2065 (2011)

6. Gilstrap, D., MacIntyre, N.: Patient-ventilator interactions. implications for clinical management. American journal of respiratory and critical care medicine 188(9), 10581068 (2013)

7. Murias, G., Villagra, A., Blanch, L.: Patient-ventilator dyssynchrony during assisted invasive mechanical ventilation. Minerva anestesiologica 79(4), 434-444 (2013)

8. Vitacca, M., Bianchi, L., Zanotti, E., Vianello, A., Barbano, L., Porta, R., Clini, E.: Assessment of physiologic variables and subjective comfort under different levels of pressure support ventilation. CHEST Journal 126(3), 851-859 (2004)

9. Schmidt, M., Banzett, R.B., Raux, M., Morélot-Panzini, C., Dangers, L., Similowski, T., Demoule, A.: Unrecognized suffering in the icu: addressing dyspnea in mechanically ventilated patients. Intensive care medicine 40(1), 1-10 (2014)

10. De Wit, M., Miller, K.B., Green, D.A., Ostman, H.E., Gennings, C., Epstein, S.K.: Ineffective triggering predicts increased duration of mechanical ventilation*. Critical care medicine 37(10), 2740-2745 (2009)

11. Hansen-Flaschen, J.H., Brazinsky, S., Basile, C., Lanken, P.N.: Use of sedating drugs and neuromuscular blocking agents in patients requiring mechanical ventilation for respiratory failure: a national survey. Jama 266(20), 2870-2875 (1991)

12. de Wit, M., Pedram, S., Best, A.M., Epstein, S.K.: Observational study of patientventilator asynchrony and relationship to sedation level. Journal of critical care 24(1), $74-80$ (2009) 
13. Shehabi, Y., Chan, L., Kadiman, S., Alias, A., Ismail, W.N., Tan, M.A.T.I., Khoo, T.M., Ali, S.B., Saman, M.A., Shaltut, A., et al.: Sedation depth and long-term mortality in mechanically ventilated critically ill adults: a prospective longitudinal multicentre cohort study. Intensive care medicine 39(5), 910-918 (2013)

14. Levine, S., Nguyen, T., Taylor, N., Friscia, M.E., Budak, M.T., Rothenberg, P., Zhu, J., Sachdeva, R., Sonnad, S., Kaiser, L.R., et al.: Rapid disuse atrophy of diaphragm fibers in mechanically ventilated humans. New England Journal of Medicine 358(13), 1327-1335 (2008)

15. Jaber, S., Jung, B., Matecki, S., Petrof, B.J.: Clinical review: ventilator-induced diaphragmatic dysfunction-human studies confirm animal model findings. Crit Care $\mathbf{1 5}(2), 206(2011)$

16. Kallet, R.H.: Patient-ventilator interaction during acute lung injury, and the role of spontaneous breathing: part 1: respiratory muscle function during critical illness. Respiratory care 56(2), 181-189 (2011)

17. Blanch, L., Villagra, A., Sales, B., Montanya, J., Lucangelo, U., Luján, M., GarcíaEsquirol, O., Chacón, E., Estruga, A., Oliva, J.C., et al.: Asynchronies during mechanical ventilation are associated with mortality. Intensive care medicine 41(4), 633-641 (2015)

18. Kondili, E., Prinianakis, G., Georgopoulos, D.: Patient-ventilator interaction. British Journal of Anaesthesia 91(1), 106-119 (2003)

19. Colombo, D., Cammarota, G., Alemani, M., Carenzo, L., Barra, F.L., Vaschetto, R. Slutsky, A.S., Della Corte, F., Navalesi, P.: Efficacy of ventilator waveforms observation in detecting patient-ventilator asynchrony*. Critical care medicine 39(11), 2452-2457 (2011)

20. Mauri, T., Yoshida, T., Bellani, G., Goligher, E.C., Carteaux, G., Rittayamai, N., Mojoli, F., Chiumello, D., Piquilloud, L., Grasso, S., et al.: Esophageal and transpulmonary pressure in the clinical setting: meaning, usefulness and perspectives. Intensive care medicine 42(9), 1360-1373 (2016)

21. Akoumianaki, E., Maggiore, S.M., Valenza, F., Bellani, G., Jubran, A., Loring, S.H., Pelosi, P., Talmor, D., Grasso, S., Chiumello, D., et al.: The application of esophageal pressure measurement in patients with respiratory failure. American journal of respiratory and critical care medicine 189(5), 520-531 (2014)

22. Sinderby, C., Liu, S., Colombo, D., Camarotta, G., Slutsky, A.S., Navalesi, P., Beck, J.: An automated and standardized neural index to quantify patient-ventilator interaction. Critical Care 17(5), R239 (2013)

23. Jansen, D., Jonkman, A.H., Roesthuis, L., Gadgil, S., van der Hoeven, J.G., Scheffer, G.J.J., Girbes, A., Doorduin, J., Sinderby, C.S., Heunks, L.M.: Estimation of the diaphragm neuromuscular efficiency index in mechanically ventilated critically ill patients. Critical Care 22(1), 238 (2018)

24. Barwing, J., Ambold, M., Linden, N., Quintel, M., Moerer, O.: Evaluation of the catheter positioning for neurally adjusted ventilatory assist. Intensive care medicine 35(10), 1809-1814 (2009)

25. Gama de Abreu, M., Belda, F.J.: Neurally adjusted ventilatory assist: letting the respiratory center take over control of ventilation. Intensive Care Medicine 39(8), 1481-1483 (2013). DOI 10.1007/s00134-013-2953-5. URL https://doi.org/10.1007/ s00134-013-2953-5

26. Vaporidi, K., Babalis, D., Chytas, A., Lilitsis, E., Kondili, E., Amargianitakis, V., Chouvarda, I., Maglaveras, N., Georgopoulos, D.: Clusters of ineffective efforts during mechanical ventilation: impact on outcome. Intensive care medicine 43(2), 184-191 (2017)

27. Blanch, L., Sales, B., Montanya, J., Lucangelo, U., Garcia-Esquirol, O., Villagra, A., Chacon, E., Estruga, A., Borelli, M., Burgueño, M.J., et al.: Validation of the Better Care ${ }^{\circledR}$ system to detect ineffective efforts during expiration in mechanically ventilated patients: a pilot study. Intensive care medicine 38(5), 772-780 (2012)

28. Younes, M., Brochard, L., Grasso, S., Kun, J., Mancebo, J., Ranieri, M., Richard, J.C. Younes, H.: A method for monitoring and improving patient: ventilator interaction. Intensive care medicine 33(8), 1337-1346 (2007)

29. Lucangelo, U., Fabris, F., Bortolussi, L., Casagrande, A., Borelli, M., Quintavalle, F.: "Apparatus To Identify Respiratory Asynchronies In An Assisted Breathing Machine" (No. EP 3308819 (A1)), April 18, 2018 (2018). URL https://lens.org/ 009-096-220-965-258 
30. Campbell, E.J.M.: The respiratory muscles and the mechanics of breathing. Lloyd-Luke (1958)

31. Cabello, B., Mancebo, J.: Work of breathing. In: Applied Physiology in Intensive Care Medicine 1, pp. 11-14. Springer (2012)

32. Oliphant, T.: NumPy: A guide to NumPy. USA: Trelgol Publishing (2006-). URL http://www.numpy.org/

33. McKinney, W.: Data Structures for Statistical Computing in Python. In: S. van der Walt, J. Millman (eds.) Proceedings of the 9th Python in Science Conference, pp. 51-56 (2010)

34. Mckinney, W.: pandas: a Foundational Python Library for Data Analysis and Statistics. Python High Performance Science Computer (2011)

35. Pedregosa, F., Varoquaux, G., Gramfort, A., Michel, V., Thirion, B., Grisel, O., Blondel, M., Prettenhofer, P., Weiss, R., Dubourg, V., Vanderplas, J., Passos, A., Cournapeau, D., Brucher, M., Perrot, M., Duchesnay, E.: Scikit-learn: Machine learning in Python. Journal of Machine Learning Research 12, 2825-2830 (2011)

36. Buitinck, L., Louppe, G., Blondel, M., Pedregosa, F., Mueller, A., Grisel, O., Niculae, V., Prettenhofer, P., Gramfort, A., Grobler, J., Layton, R., VanderPlas, J., Joly, A. Holt, B., Varoquaux, G.: API design for machine learning software: experiences from the scikit-learn project. In: ECML PKDD Workshop: Languages for Data Mining and Machine Learning, pp. 108-122 (2013)

37. scikit-learn's Authors, Contributors: scikit-learn User Manual (2018 (accessed May 5, 2019)). URL https://scikit-learn.org/stable/user_guide.html

38. Borağan Aruoba, S., Fernández-Villaverde, J.: A comparison of programming languages in macroeconomics. Journal of Economic Dynamics and Control 58, 265-273 (2015). DOI https://doi.org/10.1016/j.jedc.2015.05.009 